Review Article

\title{
Prognostic Value of EZH2 in Non-Small-Cell Lung Cancers: A Meta-Analysis and Bioinformatics Analysis
}

\author{
Kui Fan, ${ }^{1}$ Chuan-long Zhang, ${ }^{1}$ Yuan-fu Qi, ${ }^{2}$ Xin Dai, ${ }^{2}$ Yoann Birling, ${ }^{3}$ Zhao-feng Tan, ${ }^{2}$ \\ and Fang Cao ${ }^{2}{ }^{2}$ \\ ${ }^{1}$ College of Traditional Chinese Medicine, Shandong University of Traditional Chinese Medicine, Jinan, Shandong, China \\ ${ }^{2}$ Department of Oncology, Affiliated Hospital of Shandong University of Traditional Chinese Medicine, Jinan, Shandong, China \\ ${ }^{3}$ NICM Health Research Institute, Western Sydney University, Westmead, Australia
}

Correspondence should be addressed to Fang Cao; sdcaofang@163.com

Received 10 April 2020; Revised 20 September 2020; Accepted 22 October 2020; Published 9 November 2020

Academic Editor: Peyman Björklund

Copyright (C) 2020 Kui Fan et al. This is an open access article distributed under the Creative Commons Attribution License, which permits unrestricted use, distribution, and reproduction in any medium, provided the original work is properly cited.

\begin{abstract}
Background. The prognosis of non-small-cell lung cancer (NSCLC) has not been significantly improved. In the past several years, research on epigenetics is in full swing. There is a focus on the gene EZH2; however, its role as a predictor of the prognosis of NSCLC is in the debate. Objective. To clarify if the expression level of EZH2 can influence the prognosis of NSCLC and explain its prognostic value. Methods. We have systematically searched PubMed, Web of Science, and Cochrane library, screened relevant articles, and conducted a meta-analysis on the expression level of EZH2 in NSCLC. We collected the hazard ratio (HR) and the 95\% confidence interval (CI) and used STATA 12.0 to calculate the combined result of EZH2 overall survival. In addition, we conducted subgroup analyses, a sensitivity analysis, and a funnel plot to test the reliability of the results. We further validated these meta-analysis results using the Kaplan-Meier plotter database and The Cancer Genome Atlas (TCGA) database. In addition, we have investigated the correlation between EZH2 expression and EGFR expression, KRAS expression, BRAF expression, and smoking in TCGA database to further explore the mechanism behind the influence of high EZH2 expression on lung cancer prognosis. Results. 13 studies including 2180 participants were included in the meta-analysis. We found that high expression of EZH2 indicates a poor prognosis of NSCLC ( $\mathrm{HR}=1.65$ and 95\% CI 1.16-2.35; $p \leq 0.001)$. Subgroup analyses showed high heterogeneity in stages I-IV $\left(I^{2}=85.1 \%\right.$ and $\left.p \leq 0.001\right)$ and stages I-III $\left(I^{2}=66.9 \%\right.$ and $\left.p=0.029\right)$ but not in stage I $\left(I^{2}=0.00 \%\right.$ and $\left.p=0.589\right)$. In the Kaplan-Meier plotter database, there was a high expression in 963 cases and low expression in 964 cases $(\mathrm{HR}=1.31$ and $95 \% \mathrm{CI} 1.15-1.48 ; p<0.05)$. Further analysis found that the high expression of EZH2 was statistically significant in lung adenocarcinoma ( $\mathrm{HR}=1.27$ and $95 \%$ CI 1.01-1.6; $p=0.045)$, but not in lung squamous cell carcinoma ( $\mathrm{HR}=1.03$ and $95 \% \mathrm{CI} 0.81-1.3 ; p=0.820)$. The results of the TCGA database showed that the expression of EZH2 in normal tissues was lower than that in lung cancer tissues $(p<0.05)$. Smoking was associated with high expression of EZH2 $(p<0.001)$. EZH2 was also highly expressed in lung cancers with positive KRAS expression, and the correlation was positive in lung adenocarcinoma $(r=0.3129$ and $p<0.001)$. The correlation was also positive in lung squamous cell carcinoma $(r=0.3567$ and $p<0.001)$. EZH2 expression was positively correlated with BRAF expression $(r=0.2397$ and $p<0.001)$, especially in lung squamous cell carcinoma $(r=0.3662$ and $p<0.001)$. In lung squamous cell carcinoma, a positive yet weak correlation was observed between EZH2 expression and EGFR expression $(r=0.1122$ and $p<0.001)$. Conclusions. The high expression of EZH2 indicates a poor prognosis of NSCLC, which may be related to tumor stage or cancer type. EZH2 may be an independent prognostic factor for NSCLC. EZH2 high expression or its synergistic action with KRAS and BRAF mutations affects the prognosis of non-small-cell lung cancer.
\end{abstract}

\section{Introduction}

Lung cancer is the cancer that has the highest incidence and mortality rate worldwide $[1,2]$. NSCLC accounts for $80 \%$ to
$85 \%$ of all lung cancer cases [3]. In recent years, great progress in the diagnosis and treatment of NSCLC has been achieved, yet the overall five-year survival rate is still less than $21 \%$ [4]. A lot of evidence shows that epigenetic regulation 

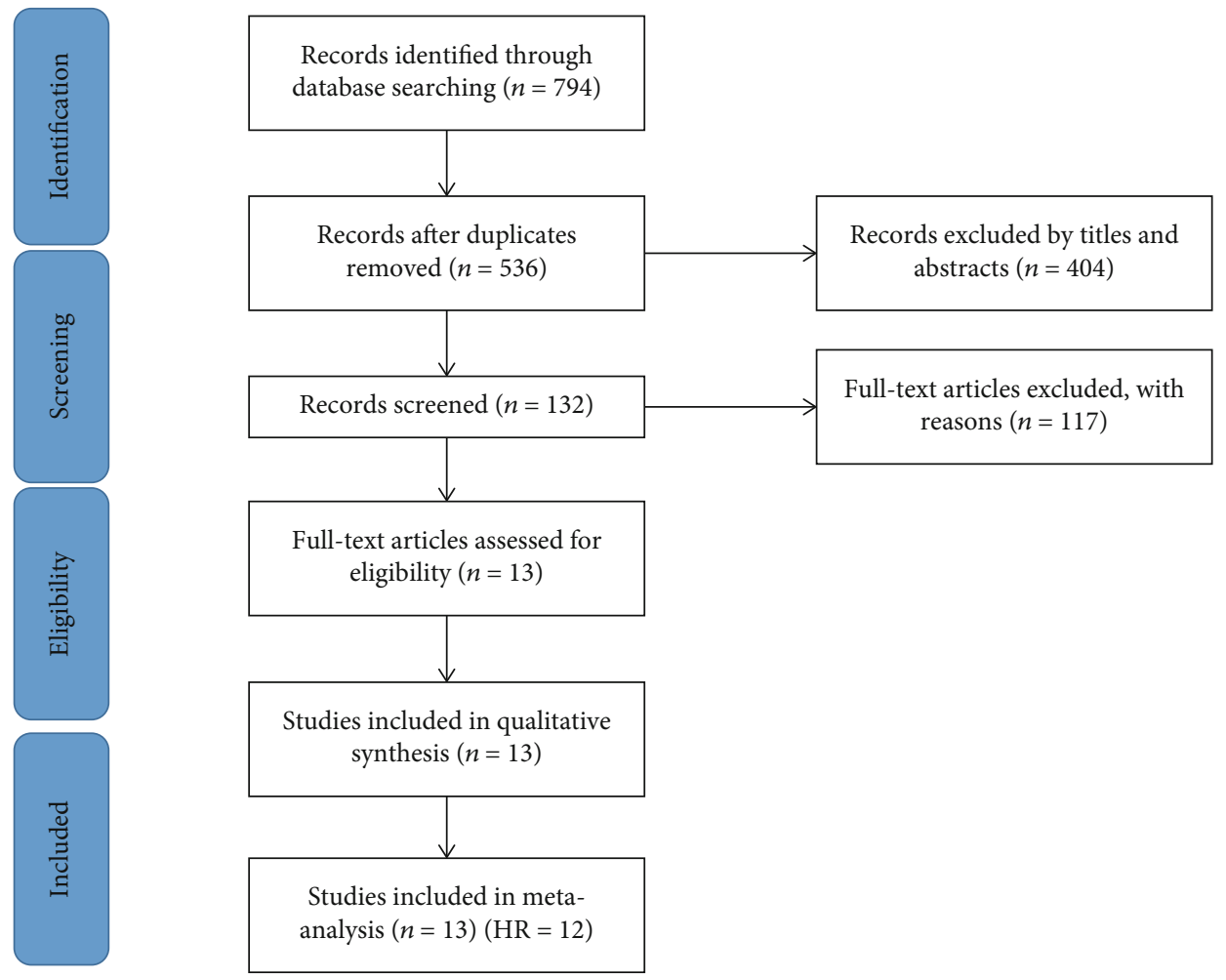

FIGURE 1: Flow diagram of the study selection process. HR: hazard ratio.

plays an important role in the occurrence and development of tumors. Epigenetic modification can regulate chromatin status and gene expression through pathways such as DNA methylation and demethylation, histone modification, and chromatin remodeling without changing the DNA sequence $[5,6]$. Enhancer of zeste homolog $2(\mathrm{EZH} 2)$ is an evolutionarily conserved gene that participates in a variety of biological functions (e.g., cell cycle, cell proliferation, and cell differentiation). EZH2 is a key factor of tumor growth and metastasis [7-9].

Recent studies suggest that EZH2 has a potential prognostic role in patients with NSCLC. However, based on their results, the prognostic value of EZH2 expression levels in NSCLC is controversial. It is generally believed that metaanalysis is a powerful statistical tool that can overcome the limitations of different individual research sample sizes and produce the best estimates. Therefore, we collected all eligible published studies and performed a meta-analysis to quantify the prognostic value of pretreatment EZH2 in NSCLC.

\section{Materials and Methods}

2.1. Search Strategy. We conducted a meta-analysis according to the PRISMA guidelines [10]. We searched the online databases PubMed, Web of Science, and Cochrane Library for every eligible study until February 2020.

The search terms were "Enhancer of zeste homolog 2" OR "EZH2" OR "ENX-1" AND "lung cancer" OR "lung adenocarcinoma" OR "lung tumors" OR "NSCLC" OR "LAD" OR "ADC." We identified duplicates using the authors' name, institution, clinical trial registry number, the number of participants, and baseline data. For the studies reported by the same author multiple times, we choose the latest and most complete publication. In addition, we manually searched previous reviews and reference lists of the articles included in our study to find other related studies. Figure 1 shows a flowchart of the article selection process.

2.2. Data Extraction. Each study was revised by two reviewers (CLZ and KF) using Endnote (Vision X9). We used the following inclusion criteria: (1) study on the relationship between EZH2 and the prognosis of NSCLC; (2) publication details available, including disease name, year of publication, and name of the first author; (3) using immunohistochemistry (IHC) or polymerase chain reaction (PCR) to detect the expression of EZH2 in lung cancer tissue; (4) investigating the relationship between EZH2 expression and survival and providing sufficient data to estimate the hazard ratios (HRs) of the survival rate and its 95\% confidence interval (CI); (5) the full text is available. If HRs cannot be obtained directly from the original study, the data were calculated using Kaplan-Meier curves according to the method provided by Tierney et al. [11]. Studies were excluded if meeting one of the exclusion criteria: (1) reviews, case reports, conference abstracts, or letter or author corrections to the editor; (2) duplicate articles; (3) animal studies; (4) survival data missing or impossible to calculate. The differences were resolved through discussions with a third investigator (FC). Data collection was conducted using a standardized extraction table.

2.3. Quality Assessment. All selected articles were evaluated using the Newcastle-Ottawa scale. This scale includes three 


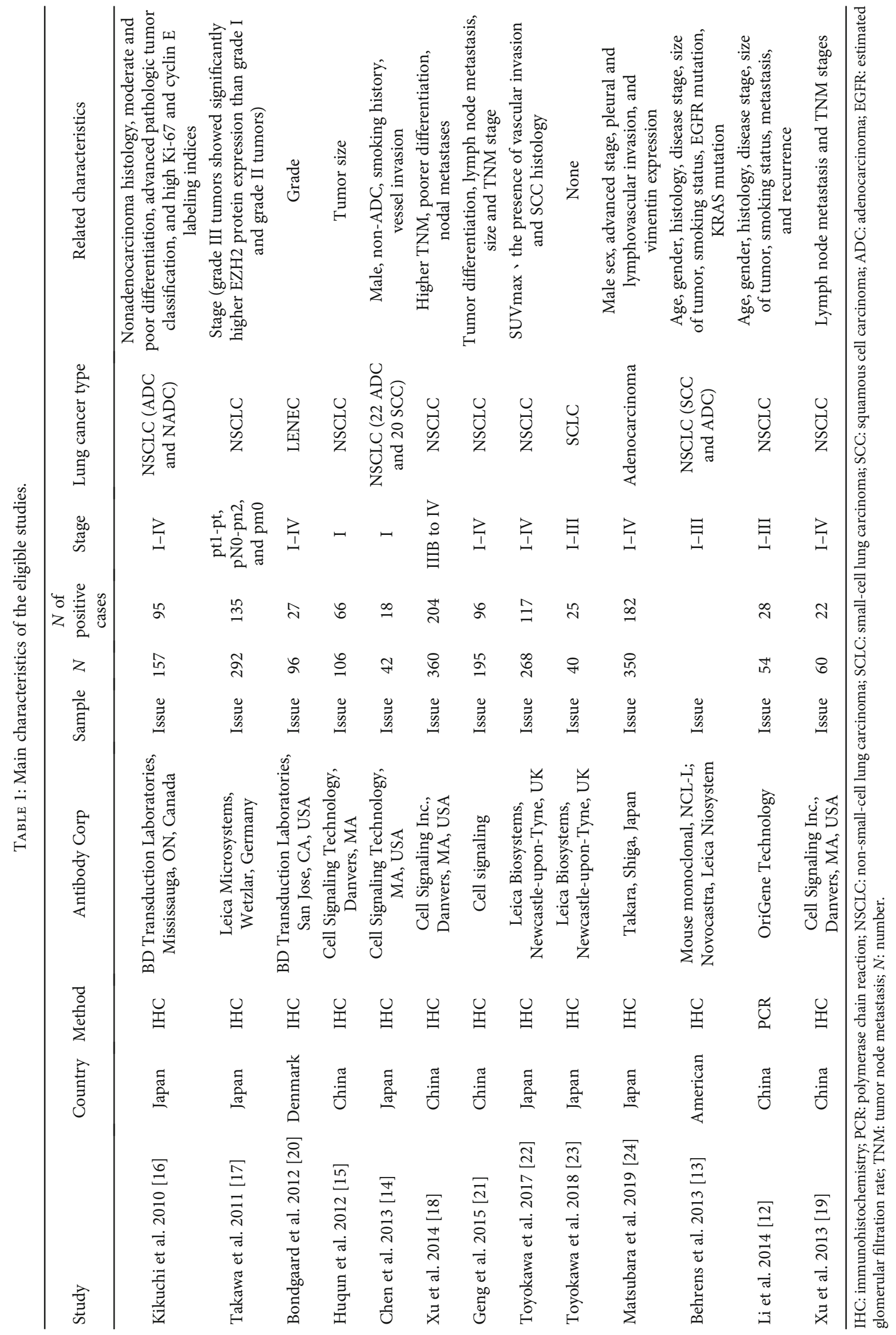


aspects: study group selection, study group comparison, and outcome measurement and has a score ranging between 0 and 9.

2.4. Bioinformatics Analysis. To further validate and complement this meta-analysis, we analyzed the relationship between EZH2 mRNA expression and NSCLC overall survival (OS) using the Kaplan-Meier plotter database. The Cancer Genome Atlas (TCGA) data of EZH2 transcriptome expression were extracted from TCGA data portal of lung adenocarcinoma and lung squamous cell carcinoma. In addition, we investigated the correlation between EZH2 expression and EGFR gene, KRAS gene, BRAF gene, and smoking in lung cancer patients from TCGA database. The results were considered statistically significant if the $p$ value was less than 0.05 .

2.5. Statistical Analysis. We used Cochran's $Q$ test and Higgin's $I^{2}$ statistics to evaluate the heterogeneity between pooled studies. If there was no significant heterogeneity ( $p>0.05$ and $I^{2}<50 \%$ ), we would like to use a fixed model; otherwise, we preferred to use a random-effects model. Publication bias was evaluated using funnel plots, Begg's statistical test, and Egger's statistical test. We used the STATA software for statistical analysis (STATA Corporation, College Station, USA, version 12.0).

\section{Result}

3.1. Study Characteristics. We retrieved 794 records from the online database using the retrieval strategy provided above. By manually reviewing the titles and abstracts, we rejected duplicates and unavailable articles. Then, we selected 132 articles for full-text screening. Finally, 13 studies were included after cross-reference. These studies were conducted in China, the United States, Denmark, and Japan, and all is aimed at assessing the relationship between EZH2 expression and prognosis in patients with NSCLC. In these studies, $\mathrm{EZH} 2$ expression was detected in lung cancer tissues in 2180 cases, including 1064 cases in which EZH2 was highly expressed. The expression of EZH2 was tested by immunohistochemical staining in 12 studies and by a polymerase chain reaction in one study [12]. The HR and 95\% CI were obtained directly from the report for six studies [13-18] and obtained from the survival curve for six other studies [12, 19-23]. The remaining study was included, but the HR could not be obtained; therefore, it was analyzed qualitatively only [19]. The characteristics of the included studies are shown in Table 1, and the HR and 95\% CI are shown in Table 2.

3.2. Qualitative Assessment. The research quality score, as measured with the Newcastle-Ottawa scale, was above 6 for all the studies (Table 3).

3.3. Meta-Analysis Results. Survival analysis was performed with 12 studies we included. Because of the high degree of heterogeneity $\left(I^{2}=76.3 \%\right.$ and $\left.p \leq 0.001\right)$, we chose a random-effects model to compare the low and high expression of EZH2. We found that a high expression of EZH2 indi-
TABLE 2: Summary of HRs and their 95\% CI

\begin{tabular}{lcccc}
\hline Author & Year & HR & LCI & UCI \\
\hline Kikuchi et al. [16] & 2010 & 2.08 & 1.32 & 3.23 \\
Takawa et al. [17] & 2011 & 1.482 & 1.051 & 2.091 \\
Bondgaard et al. [20] & 2012 & 0.28 & 0.13 & 0.57 \\
Huqun et al. [15] & 2012 & 2.8 & 1.19 & 6.59 \\
Chen et al. [14] & 2013 & 1.975 & 0.775 & 5.031 \\
Xu et al. [19] & 2014 & 4.32 & 2.13 & 8.73 \\
Geng et al. [21] & 2015 & 2.39 & 1.66 & 3.46 \\
Toyokawa et al. [22] & 2017 & 2.28 & 1.02 & 5.09 \\
Toyokawa et al. [23] & 2018 & 0.57 & 0.16 & 2.01 \\
Matsubara et al. [24] & 2019 & 2.64 & 1.41 & 4.94 \\
Behrens et al. [13] & 2013 & 1.943 & 1.387 & 2.723 \\
Li et al. [12] & 2014 & 0.45 & 0.15 & 1.35 \\
\hline
\end{tabular}

CI: confidence interval; HR: hazard ratio; LCI: low confidence interval; UCI: upper confidence interval.

cated poor OS $(\mathrm{HR}=1.65$ and $95 \%$ CI $1.16-2.35 ; p \leq 0.001)$ (Figure 2(a)). To find sources of heterogeneity between the studies, we performed subgroup analyses. Subgroup analyses showed a high heterogeneity in stages I-IV $\left(I^{2}=85.1 \%\right.$ and $p \leq 0.001)$ and in stages I-III $\left(I^{2}=66.9 \%\right.$ and $\left.p=0.029\right)$ (Figure 2(b)). There was no significant heterogeneity in stage I $\left(I^{2}=0.00 \%\right.$ and $\left.p=0.589\right)$. These results suggest that the cancer stage may be a source of heterogeneity. The study that was not included in the meta-analysis [19] also showed that the regulation of EZH2 expression is upregulated in lung cancer and that its expression is positively correlated with cancer stage and lymph node metastasis in patients with lung cancer.

3.4. Survival Analysis of Lung Cancer through Kaplan-Meier Plotter Database. In order to verify the results of the metaanalysis, we chose to use the Kaplan-Meier database for survival analysis. Among the NSCLC medical records included in the study, EZH2 mRNA had a high expression in 962 cases with a median survival time of 54.17 months and a low expression in 964 cases with a median survival time of 79.50 months $(\mathrm{HR}=1.31$ and $95 \%$ CI $1.15 \sim 1.48 ; p<0.05)$ (Figure 3(a)). Further analysis showed that 357 cases of lung adenocarcinoma with EZH2 high expression had a median survival time of 90 months and 360 cases with EZH2 with low expression had a median survival time of 119.87 months $(\mathrm{HR}=1.27$ and $95 \%$ CI 1.01-1.6; $p<0.05$ ) (Figure 3(b)). In 261 cases of lung squamous cell carcinoma, EZH2 mRNA had a high expression with a median survival time of 52.97 months, and in 263 cases, there was a low expression with a median survival time of 62.00 months $(\mathrm{HR}=1.03$ and $95 \%$ CI $0.81-1.3 ; p=0.82$ ) (Figure $3(\mathrm{c})$ ). Therefore, most of the results from the Kaplan-Meier plotter database analysis are consistent with our meta-analysis; nonetheless, no significant statistical significance was found for lung squamous cell carcinoma. This may suggest that the expression of EZH2 affects the prognosis of patients with lung cancer, and this relation may depend on the pathological type. 
TABLE 3: Quality assessment based on the Newcastle-Ottawa scale.

\begin{tabular}{|c|c|c|c|c|c|}
\hline Study & Year & Selection & Comparability & Outcome & Total score \\
\hline Kikuchi et al. [16] & 2010 & 4 & 2 & 2 & 8 \\
\hline Takawa et al. [17] & 2011 & 3 & 2 & 2 & 7 \\
\hline Bondgaard et al. [20] & 2012 & 4 & 1 & 2 & 7 \\
\hline Huqun et al. [15] & 2012 & 3 & 2 & 2 & 7 \\
\hline Chen et al. [14] & 2013 & 3 & 1 & 3 & 7 \\
\hline Xu et al. [19] & 2013 & 4 & 2 & 3 & 9 \\
\hline Behrens et al. [13] & 2013 & 4 & 2 & 2 & 8 \\
\hline $\mathrm{Xu}$ et al. [18] & 2014 & 4 & 1 & 2 & 7 \\
\hline Li et al. [12] & 2014 & 3 & 1 & 2 & 6 \\
\hline Geng et al. [21] & 2015 & 3 & 2 & 2 & 7 \\
\hline Toyokawa et al. [22] & 2017 & 3 & 2 & 2 & 7 \\
\hline Toyokawa et al. [23] & 2018 & 3 & 2 & 2 & 7 \\
\hline Matsubara et al. [24] & 2019 & 3 & 2 & 2 & 7 \\
\hline
\end{tabular}

3.5. EZH2 Expression Analysis through TCGA Database. We selected transcriptome analysis data from patients with lung adenocarcinoma and lung squamous cell carcinoma from TCGA. After data integration, 108 normal specimens and 1037 lung cancer specimens were extracted. The expression of EZH2 and the correlation between the high expression of EZH2 and cancer stage between normal specimens and tumor specimens were compared. The results showed that the expression of EZH2 in lung cancer tissues was significantly different from that in normal tissues $(p<0.05)$ (Figure $4(\mathrm{a}))$, and there was a significant correlation between EZH2 expression and the cancer stage $(p<0.05)$ (Figure $4(\mathrm{~b}))$.

We extracted from TCGA database from lung adenocarcinoma patients (among which 165 were nonsmokers and 361 were smokers) and lung squamous carcinoma patients (among which 86 were nonsmokers and 464 were smokers), 526 lung adenocarcinoma patients and 550 lung squamous carcinoma patients with EGFR expression, 585 lung adenocarcinoma patients and 550 lung squamous carcinoma patients with KRAS expression, and 527 lung adenocarcinoma patients and 502 lung squamous carcinoma patients with BRAF expression. We found that the expression of EZH2 is positively correlated with KRAS $(r=0.3167$ and $p<0.001)$ and BRAF $(r=0.2397$ and $p<0.0001)$ gene expression in lung squamous cell carcinoma and lung adenocarcinoma (Figures 5(a) and 5(g)). In lung squamous cell carcinoma, EZH2 expression is positively correlated with EGFR expression, but the correlation is weak $(r=0.1122$ and $p<0.001$ ) (Figure 5(f)). TCGA data shows that high expression of EZH2 is related with smoking $(p<0.0001)$ (Figure $5(\mathrm{j}))$, especially in lung adenocarcinoma $(p=0.0011$ ) (Figure 5(k)). The result was not statistically significant in lung squamous cell carcinoma $(p=0.8453)$ (Figure 5(l)).

3.6. Sensitivity Analysis. To evaluate the stability of the metaanalysis results, we conducted a sensitivity analysis by excluding studies one by one and recalculating the combined HR. We found that no individual study affected the stability of the entire meta-analysis (Figure 6).
3.7. Publication Bias Assessment. Begg's funnel plot indicated an absence of risk of publication bias (Table 4 and Figure 7). This was confirmed by Egger's regression intercept (Table 5).

\section{Discussion}

The purpose of this study was to investigate the relationship between the expression of EZH2 and the prognosis of NSCLC and to evaluate the prognostic value of EZH2 in NSCLC. The high expression of EZH2 is closely related to a poor prognosis in many tumors, but its value in the prognosis of NSCLC is still controversial $[8,25,26]$. A total of 13 studies were included in this meta-analysis, with HR as the effect size and the 95\% CI upper and lower limits as variables. The meta-analysis showed that high expression of EZH2 in NSCLC tissues indicates a poor prognosis. The analysis of biological information mined by the Kaplan-Meier plotter database and TCGA database is consistent with this result, suggesting that EZH2 may be an independent prognostic factor for NSCLC. To our best knowledge, this is to date the only meta-analysis to estimate the survival rate of patients with NSCLC in which the correlation between EZH2 expression rate and $\mathrm{HR}$ is explored. Furthermore, previous metaanalyses did not specifically explain the prognostic relationship between EZH2 and lung cancer [27], or no bioinformatics evidence was used to support it [28]. Therefore, we searched TCGA database and Kaplan-Meier plotter database for large-scale global non-small-cell lung cancer database mining to find EZH2 potential correlation with NSCLC. The Kaplan-Meier plotter database analysis showed that the median survival time of patients with EZH2 mRNA high expression is 54.17 months, and the median survival time of patients with EZH2 mRNA low expression is 79.50 months $(\mathrm{HR}=1.31$ and $p<0.05)$, suggesting $\mathrm{EZH} 2$ high expression in NSCLC tissues indicates a poor prognosis and has significant correlation with cancer stage and pathological type. As single-stranded RNA molecules with a length of 200 to $100,000 \mathrm{nt}$, long-chain noncoding RNAs have multiple interactions with EZH2. LINC01234 interacts with RNAbinding proteins LSD1 and $\mathrm{EZH} 2$, resulting in histone 


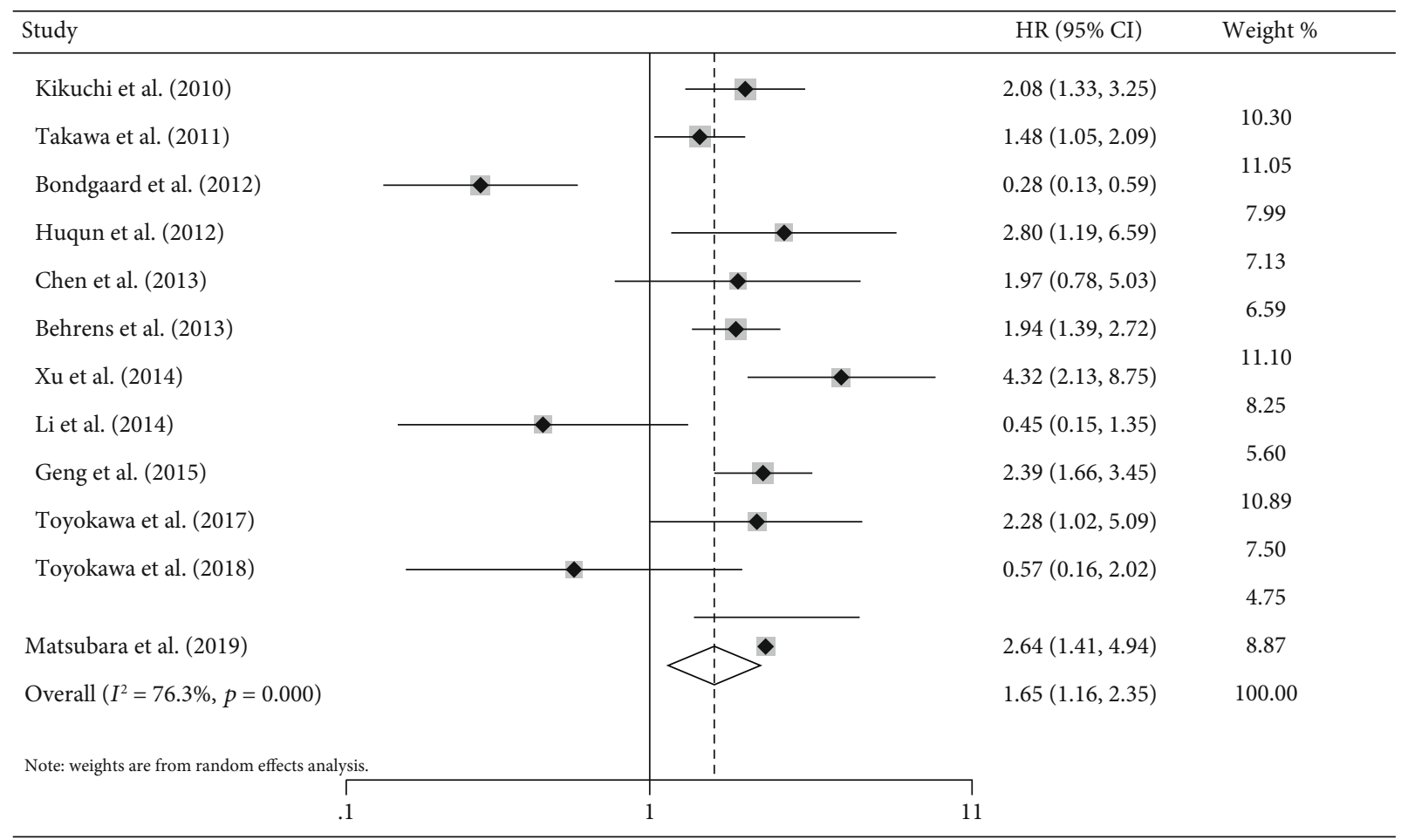

(a)

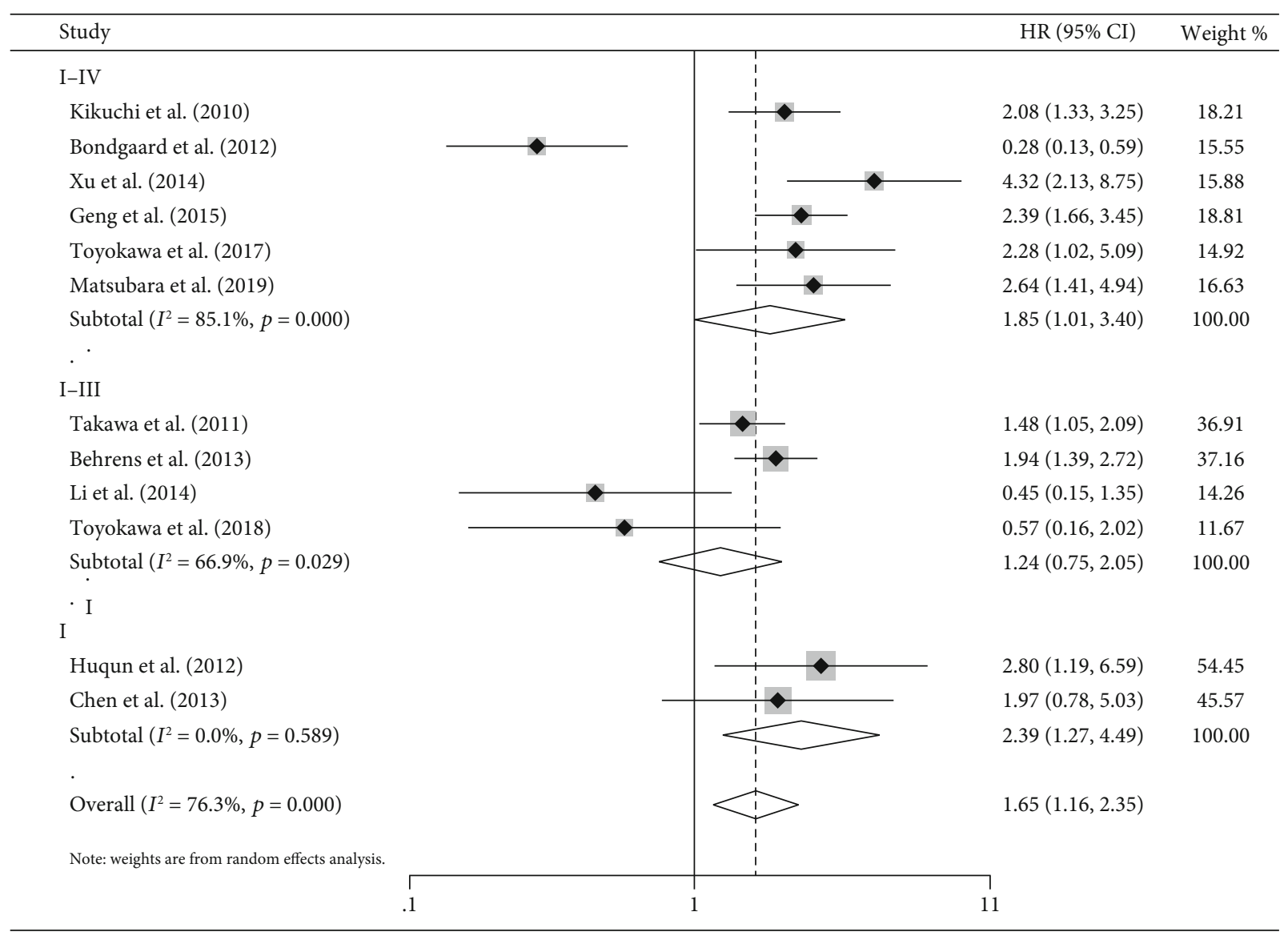

(b)

FIgURE 2: Forest plot of overall survival analysis and disease-free survival analysis. (a) Meta-analysis of EZH2 expression and overall survival. (b) Meta-analysis of EZH2 expression and overall survival in different stages of lung cancer. 


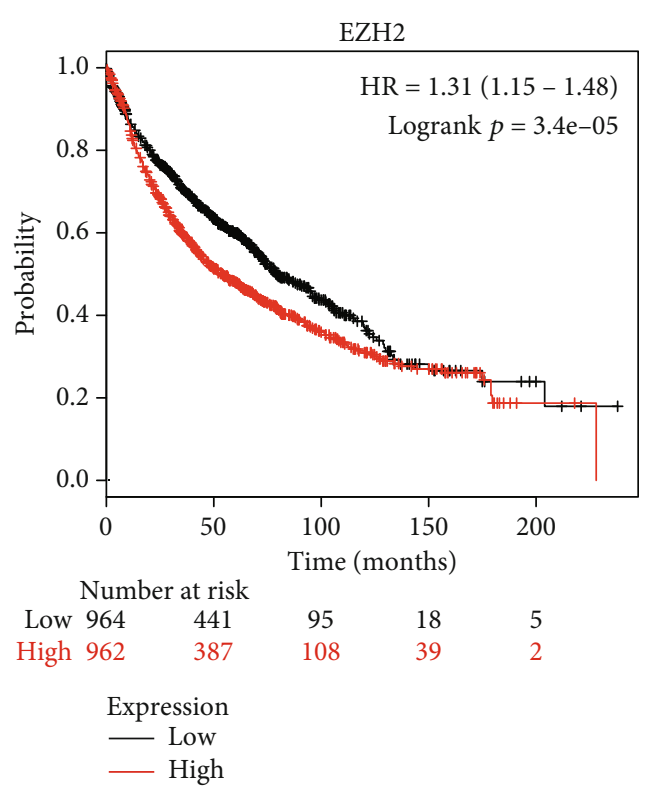

(a)

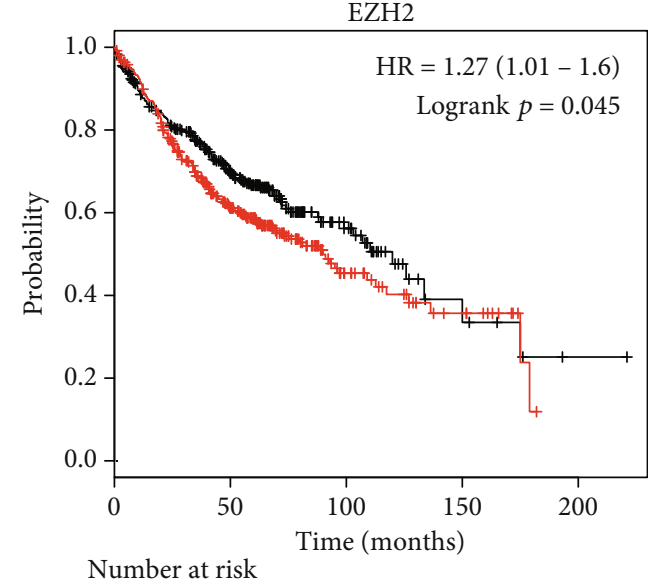

$\begin{array}{rrrrr}\text { Low } 360 & 182 & 36 & 7 & 1 \\ \text { High } 360 & 166 & 33 & 12 & 0\end{array}$
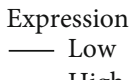

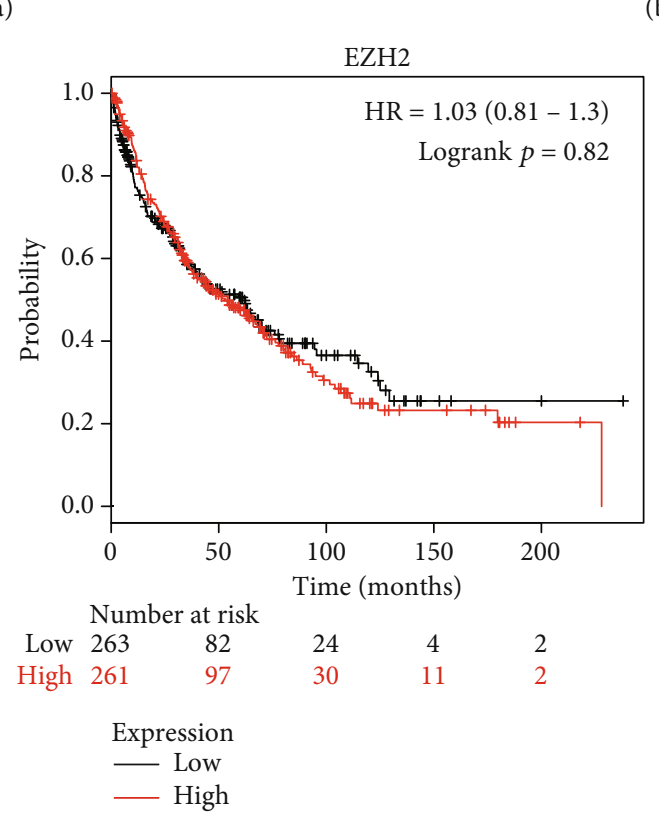

(b)

(c)

Figure 3: Kaplan-Meier survival curves for lung cancer patients, stratified by EZH2 expression levels: (a) lung cancer, (b) lung adenocarcinoma, and (c) lung squamous cell carcinoma.

modification and transcriptional suppression of the antiproliferative gene BTG2 [29]. RNA immunoprecipitation and chromatin immunoprecipitation experiments showed that LINC00467 recruited EZH2 to the htrA serine peptidase 3 (HTRA3) promoter, thereby inhibiting the expression of downstream genes of HTRA3 [30]. IncRNA FOXC2-AS1 may inhibit p15 expression by interacting with EZH2, thereby promoting the tumorigenesis of NSCLC [31]. This shows that, through the upregulation of the EZH2 expression, long noncoding RNA silences tumor suppressor genes and promotes lung cancer invasion and migration, which are associated with poor prognosis. This is consistent with our findings. However, the high expression of EZH2 is not only related to the regulation of long noncoding.

RNA, but also to other aspects of epigenetics, such as DNA methylation and histone modification. According to the human ENCODE database (https://www.encodeproject .org/), EZH2 is one of the targets of the polycomb protein and plays an important role in embryo development and tumorigenesis. EZH2 is an important candidate regulatory factor for the aging methyl group and is inhibited in senescent cells, i.e., the share of EZH2 may decrease with age $[32,33]$. This indicates that the expression of EZH2 should decrease with age. However, the data we obtained from 


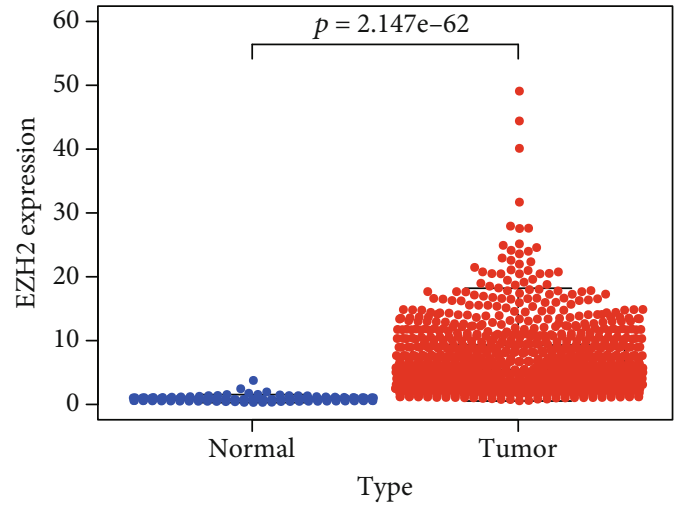

(a)

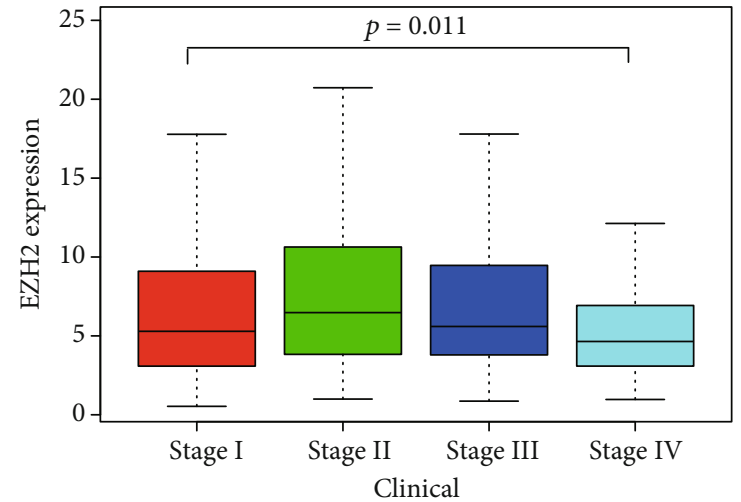

(b)

Figure 4: TCGA data analysis. (a) Comparison of EZH2 expression between normal tissue and tumor tissue. (b) Comparison of EZH2 expression between stages of patients with lung cancer.

TCGA indicates that EZH2 is highly expressed in NSCLC even in older adults [29-31, 34, 35]. This shows that the high expression of EZH2 "juvenizes" tumor cells, thereby affecting the prognosis of non-small-cell lung cancer.

The main treatment of patients with NSCLC is chemotherapy, and drug resistance is a major clinical problem. Some studies have suggested that AFAP1-AS1 can activate the PI3K/AKT pathway and interact with EZH2 to inhibit the apoptosis of A549/DDP cells, thereby inducing NSCLC resistance to DDP [36]. The specific interaction of LINC00665 and EZH2 significantly reduces the expression level of phosphorylated AKT (p-AKT) [37]. A study by Zhan et al. [38] showed that cisplatin induces high expression of HOXB13 in lung adenocarcinoma cells. HOXB13 can directly upregulate a series of genes related to metastasis and drug resistance by directly binding to $\mathrm{EZH} 2$, promoting tumor progression and predicting poor prognosis. The above studies show that EZH2 is involved in drug resistance in patients with NSCLC, which can lead to a poor prognosis. Surprisingly, EZH2 has also a certain correlation with the body's immunity, i.e., it can promote the development of $\mathrm{T}$ cells and B cells [39] and plays an important role in B cell division and activation [40]. In an experiment, it was found that a lentivirus expressing shRNA mediated EZH2 gene knockdown, inhibiting the mRNA and protein expression levels of PD-L1, thereby delaying the progression of lung cancer in vivo by enhancing the antitumor immune response. This indicates that EZH2 overexpression leads to poor prognosis in patients with lung cancer and is positively correlated with the expression of the immunosuppressive molecule PDL1 [41]. Moreover, $\mathrm{Wu}$ et al. [42] experimentally proved that curcumin inhibits the growth and metastasis of lung cancer, partly by inhibiting EZH2. At present, there are many studies on EZH2 inhibitors [43-46], and some drugs have entered clinical research phase. All the above shows that EZH2 is a very promising independent prognostic factor for NSCLC.

Cancer stage and pathological type are important factors influencing the survival of patients with NSCLC [47]. Previous studies have determined the clinical and pathological characteristics of early NSCLC, such as large tumor volume, lymphatic vessel invasion (LVI), or visceral pleural invasion
(VPI) characteristics of poor prognosis [48, 49]. Our subgroup analysis also suggests that the cancer stage may be a source of heterogeneity.

The results from the Kaplan-Meier survival curve database also suggest that EZH2 in lung adenocarcinoma predicts a poor prognosis. However, the current staging system is based on anatomical information and is not comprehensive enough in predicting survival outcomes and reflecting NSCLC biological heterogeneity. Therefore, it is very valuable to study noninvasive and easily available pretreatment variables to estimate the survival outcome of lung cancer. It is necessary to further study the prognostic value of EZH2 for NSCLC.

As we all know, smoking is an important risk factors for lung cancer. As the amount of smoking increases, the prognosis of patients also deteriorates [50]. Studies have found that smoking can affect the expression of EZH2 through reduced DAB2IP via $\mathrm{H} 3 \mathrm{~K} 27 \mathrm{me} 3$ in COPD patients and promote the transformation of COPD into lung cancer [51]. We investigated the relationship between the expression of EZH2 in TCGA database and smoking. The extraction results of TCGA database showed that smoking was related to the high expression of EZH2, especially in lung adenocarcinoma. Moreover, the results of 6 studies included in the review showed that smoking is an exposure factor of EZH2 expression (Table 6). Studies have shown that exposure to tobacco smoke condensate induces increased the expression of EZH2 in cultured lung cancer cell lines [52], and DNA hypermethylation is a common risk factor for smoking behavior in NSCLC patients [53]. Therefore, the high expression of EZH2 may be related to long-term smoking.

Current treatments allow more targeted therapies and immunotherapy into our field of vision. Studies have shown that EZH2 promotes tumorigenesis by methylating the expression of multiple tumor suppressor genes [7]. In nonsmall-cell lung cancer, we found that the expression of $\mathrm{EZH} 2$ is associated with poor prognosis. We further analyzed the expression of EZH2 in EGFR, KRAS, and BRAF-positive patients. The results showed that high expression of EZH2 is often present in KRAS and BRAF-positive patients. Previous research results indicate that RAS is a key downstream 


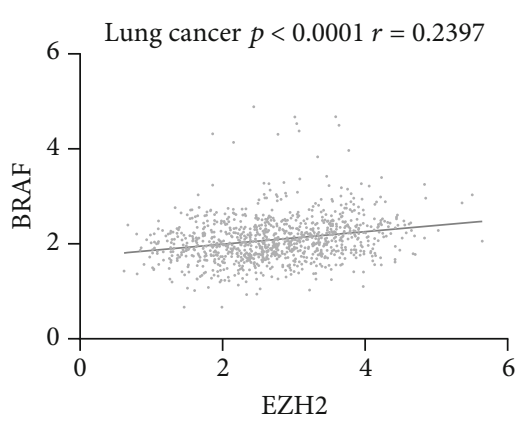

(a)

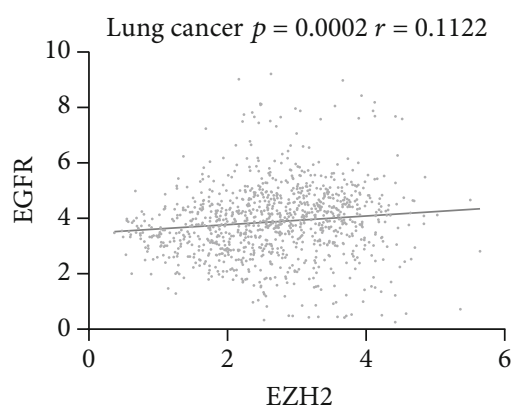

(d)

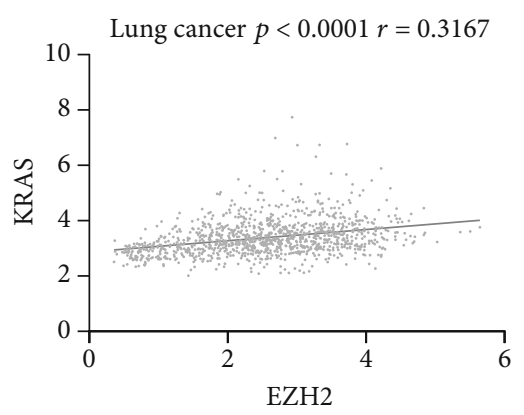

(g)

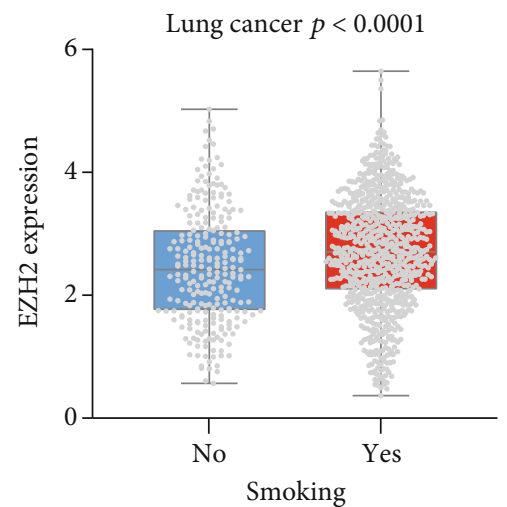

(j)

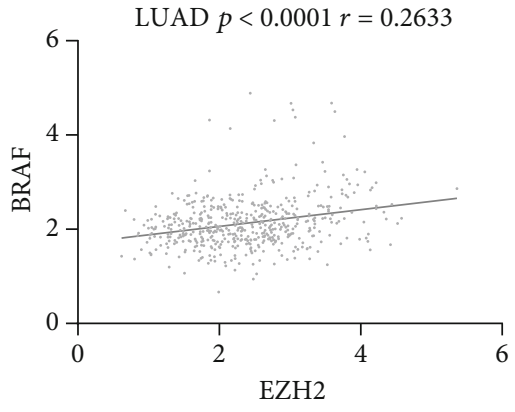

(b)

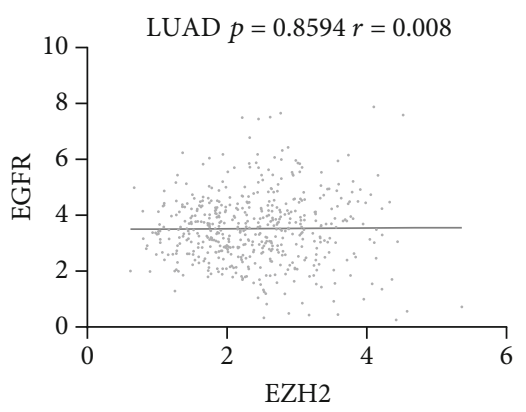

(e)

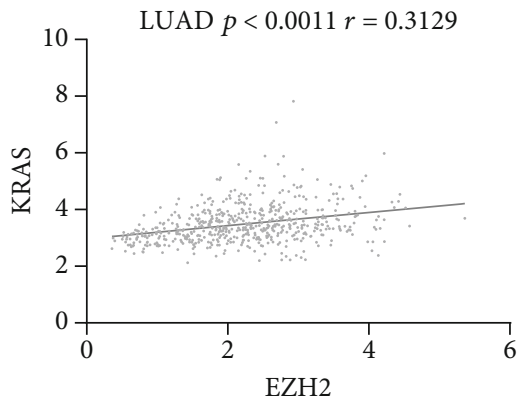

(h)

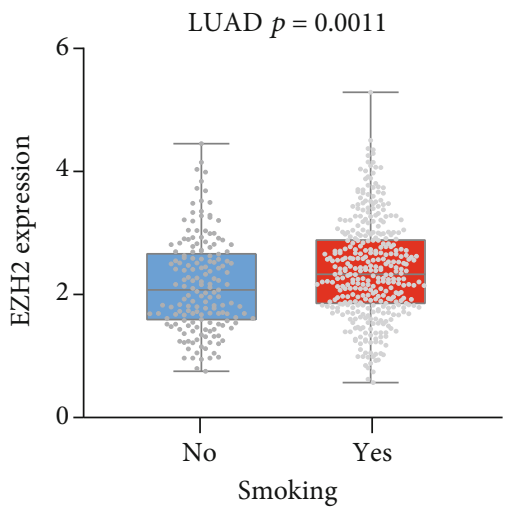

$(\mathrm{k})$

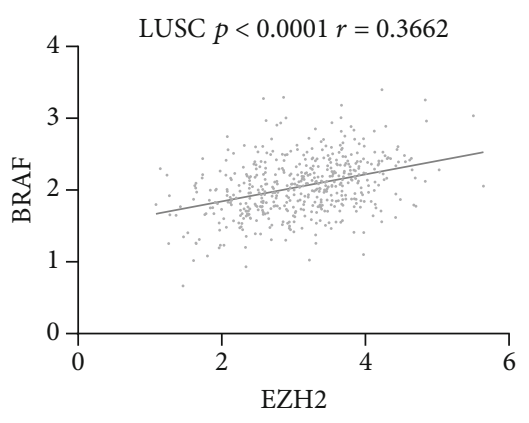

(c)

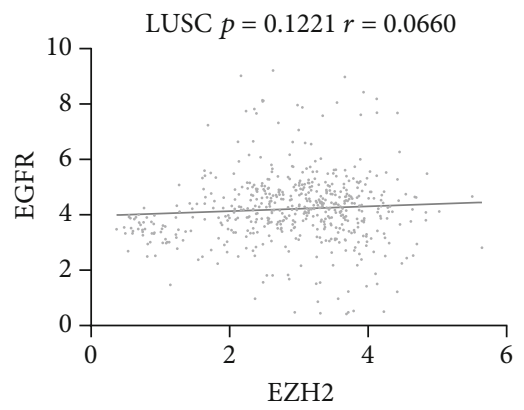

(f)

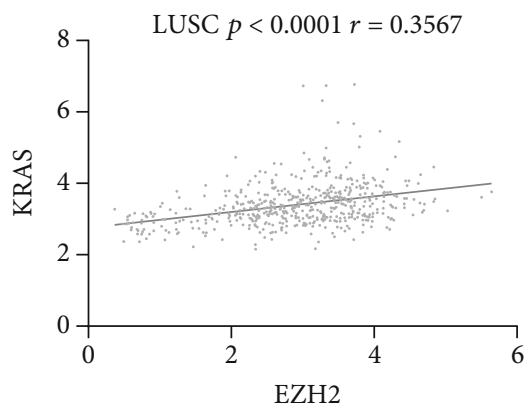

(i)

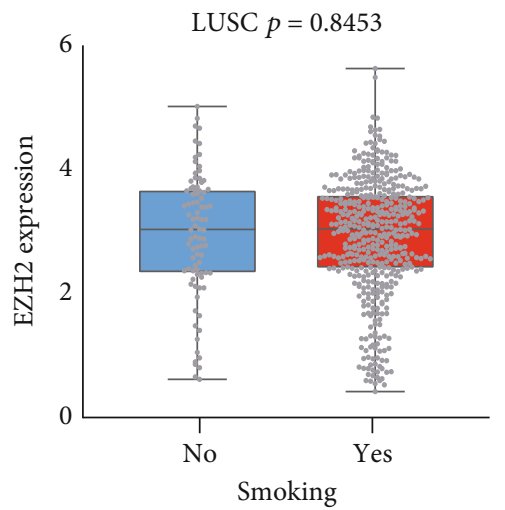

(l)

FIGURE 5: TCGA data analysis. (a) Overall correlation between EZH2 and BRAF in adenocarcinoma and squamous cell carcinoma. (b) Correlation between EZH2 and BRAF in adenocarcinoma. (c) Correlation between EZH2 and BRAF in squamous cell carcinoma. (d) Overall correlation between EZH2 and EGFR in adenocarcinoma and squamous cell carcinoma. (e) Correlation between EZH2 and EGFR in adenocarcinoma. (f) Correlation between EZH2 and EGFR in squamous cell carcinoma. (g) Overall correlation between EZH2 and KRAS in adenocarcinoma and squamous cell carcinoma. (h) Correlation between EZH2 and KRAS in adenocarcinoma. (i) Correlation between EZH2 and KRAS in squamous cell carcinoma. (j) Overall correlation between EZH2 and smoking in adenocarcinoma and squamous cell carcinoma. (k) Correlation between EZH2 and smoking in adenocarcinoma. (l) Correlation between EZH2 and smoking in squamous cell carcinoma. Lung cancer: adenocarcinoma of lung and squamous cell lung carcinoma; LUAD: adenocarcinoma of lung; LUSC: squamous cell lung carcinoma; $r$ : coefficient of correlation. 


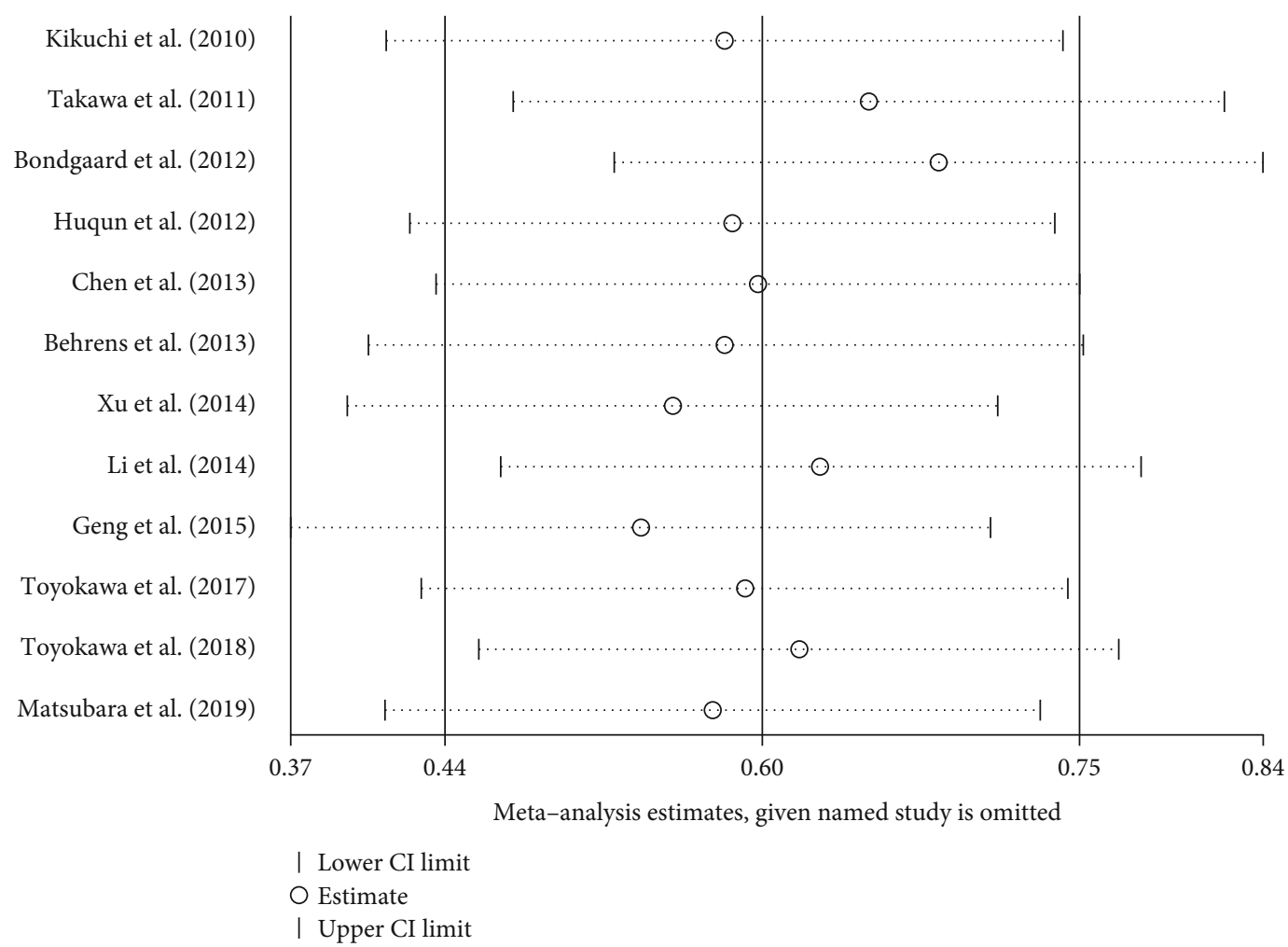

FIGURE 6: Result of sensitivity analyses by omitting one study in each turn.

TABle 4: Begg's test.

\begin{tabular}{l} 
Begg's test \\
\hline adj.Kendall s score $(P-Q)=-16$ \\
Std.Dev.of score $=14.58$ \\
Number of studies $=12$ \\
$z=-1.1$ \\
$\operatorname{Pr}>|z|=0.273$ \\
$z=1.03$ (continuity corrected) \\
$\operatorname{Pr}>|z|=0.304$ (continuity corrected) \\
\hline adj: adjusted. Std. Dev: standard deviation.
\end{tabular}

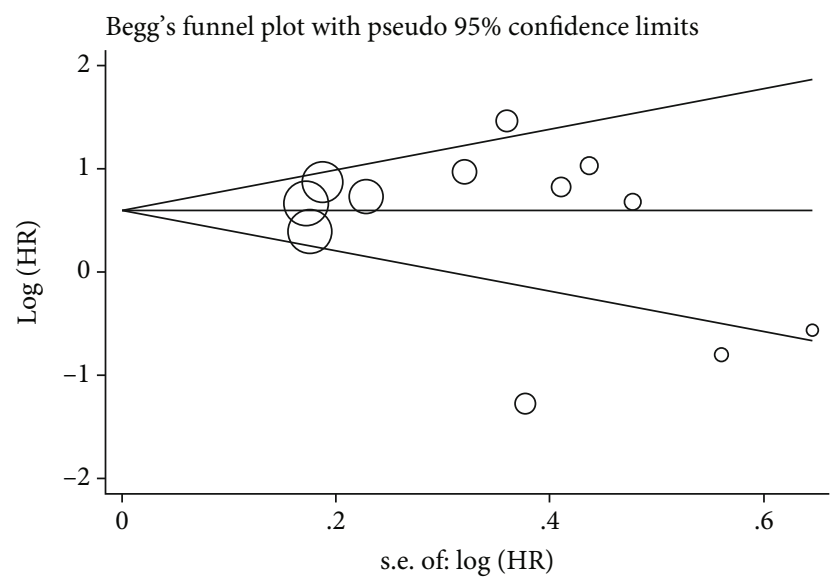

FIGURE 7: Funnel plot of EZH2 and overall survival.
TABLE 5: Egger's test.

\begin{tabular}{lcccccc}
\hline Std_Eff & Coef. & Std. err. & $t$ & $p>|t|$ & \multicolumn{2}{c}{$\begin{array}{c}\text { (95\% Conf. } \\
\text { interval) }\end{array}$} \\
\hline Slope & 0.930 & 0.401 & 2.320 & 0.043 & 0.036 & 1.824 \\
Bias & -1.331 & 1.464 & -0.910 & 0.385 & -4.592 & 1.93 \\
\hline
\end{tabular}

TABLE 6: Correlation between EZH2 and smoking in six studies.

\begin{tabular}{lccccc}
\hline \multirow{2}{*}{ Study } & \multirow{2}{*}{ OR } & \multicolumn{2}{c}{ EZH2 high } & \multicolumn{2}{c}{ EZH2 low } \\
& & Yes & No & Yes & No \\
\hline Kikuchi et al. 2010 [16] & 8.69 & 74 & 11 & 24 & 31 \\
Takawa et al. 2011 [17] & 3.64 & 114 & 21 & 94 & 63 \\
Chen et al. 2013 [14] & 4.90 & 14 & 4 & 10 & 14 \\
Geng et al. 2015 [21] & 1.02 & 50 & 46 & 51 & 48 \\
Toyokawa et al. 2018 [23] & 5.50 & 22 & 1 & 12 & 3 \\
Li et al. 2014 [12] & 0.23 & 10 & 12 & 25 & 7 \\
\hline
\end{tabular}

effector of epidermal growth factor receptor (EGFR), which is activated by mutation and/or overexpression in a variety of human cancers. B-Raf proto-oncoprotein (BRAF) is a serine/threonine kinase that regulates cell proliferation, differentiation, angiogenesis, and cell death. It plays a role in the downregulation of RAS and sends signals through the MAPK/ERK pathway [54, 55]. We believe that the mechanism of high expression of EZH2 on non-small-cell lung cancer may be related to the KRAS and BRAF pathways. It may be that EZH2 silences the genes that inhibit KRAS and BRAF 
leading to their high expression, or it may be that KRAS and BRAF promote tumor development by activating EZH2.

According to our results, the correlation between the expression of EZH2 and the expression of EGFR in lung carcinoma is not statistically significant. It means there is no evidence of correlation between the two. Nonetheless, from the perspective of its relationship with KRAS and BRAF, we may think that the mechanism or pathways of EZH2 and EGFR have some similarities, but as far as we know, no relevant studies have confirmed this. Studies have shown that the expression of EZH2 is detected in lung precancerous lesions [56], which indicates that EZH2 may also play a role in the diagnosis of lung cancer. Therefore, we believe that the combined detection of EZH2 with EGFR, KRAS, and BRAF has more advantages in the diagnosis and treatment of lung cancer. EZH2 may be a new target for the treatment of non-small-cell lung cancer. This is only speculative at this stage and further experimental verification is still needed.

$\mathrm{EZH} 2$ is not only related to NSCLC, but it is also closely related to the occurrence and development of other tumors. For example, in metastatic prostate cancer, both the content of EZH2 messenger RNA and EZH2 protein are increased. Additionally, studies show that high expression of EZH2 is related to bad prognosis in prostate cancer, and it is also an indicator to distinguish indolent prostate cancer from prostate cancer with lethal evolution [57]. Nikoloski et al. [58] pointed out that EZH2 is the target of various types of deletions and mutations in bone marrow dysplasia (MDS), and $\mathrm{EZH} 2$ may play the role of an oncogene. Wang et al. [59] found that lncRNA MALAT1 promotes the development of mantle cell lymphoma by binding to EZH2. In diffuse large B cell lymphoma, McCabe et al. [60] found that inhibition of EZH2 activity may provide a promising treatment for EZH2 mutant lymphoma. However, Schmitz et al. found that diffuse large B cell lymphomas based on EZH2 mutations and BCL2 translocation genetic subtypes have higher survival rates than other subtypes [61]. This also suggests to some extent that EZH2 not only promotes tumor progression but may play a beneficial role in certain tumors or certain tumor subtypes. Depending on the cellular environment and the activated oncogenic pathways, the changes in epigenetic modifications caused by EZH2 defects may lead to tumor progression through various mechanisms.

This is the first meta-analysis investigating the relationship between EZH2 expression and prognosis in patients with NSCLC that is supported by bioinformatics analysis. However, our study suffers from certain limitations. Firstly, we found a significant heterogeneity between the included studies $\left(I^{2}=76.3 \%\right.$ and $\left.p \leq 0.001\right)$. Despite the use of subgroup analysis and sensitivity analysis, the source of heterogeneity cannot be fully traced. Secondly, this study included 13 studies with 2180 patients, of which six had no clear HR values. The part of HRs was estimated from the Kaplan-Meier survival curve, which reduced the credibility of our results. And all the included studies were retrospective designed. Finally, this review is limited to studies published in English. Therefore, publication bias cannot be ruled out.

\section{Conclusion}

Our meta-analysis and bioinformatics analyses show that the high expression of EZH2 indicates poor OS, and EZH2 may be an independent prognostic factor affecting NSCLC. EZH2 high expression or its synergistic action with KRAS and BRAF mutations affects the prognosis of non-small-cell lung cancer. However, these results are based on retrospective studies, which limit the conclusions. Therefore, further research is needed on the effect of EZH2 high expression on the prognosis of patients with NSCLC.

\section{Conflicts of Interest}

The authors declare that they have no conflicts of interest.

\section{Authors' Contributions}

KuiFan and Chuan-longZhang are equal contributors and co-first authors. Fist authorship determined by a coin toss. All authors read and approved the final manuscript.

\section{Acknowledgments}

Study design, data collection and analysis, the decision to publish, and preparation of the manuscript were supported by the Chinese Medicine Technological Development Plan of Shandong Province (2019-0081).

\section{References}

[1] F. Bray, J. Ferlay, I. Soerjomataram, R. L. Siegel, L. A. Torre, and A. Jemal, "Global cancer statistics 2018: GLOBOCAN estimates of incidence and mortality worldwide for 36 cancers in 185 countries," CA: A Cancer Journal for Clinicians, vol. 68, no. 6, pp. 394-424, 2018.

[2] R. L. Siegel, K. D. Miller, and A. Jemal, "Cancer statistics, 2020," CA: A Cancer Journal for Clinicians, vol. 70, no. 1, pp. 7-30, 2020.

[3] V. C. Cordeiro de Lima, C. S. Baldotto, C. H. Barrios et al., "Stage III non-small-cell lung cancer treated with concurrent chemoradiation followed or not by consolidation chemotherapy: a survival analysis from a Brazilian multicentric cohort," Journal of Global Oncology, vol. 4, pp. 1-11, 2018.

[4] T. Lu, X. Yang, Y. Huang et al., "Trends in the incidence, treatment, and survival of patients with lung cancer in the last four decades," Cancer Management and Research, vol. 11, pp. 943 953, 2019.

[5] E. L. Greer and Y. Shi, "Histone methylation: a dynamic mark in health, disease and inheritance," Nature Reviews Genetics, vol. 13, no. 5, pp. 343-357, 2012.

[6] R. Margueron and D. Reinberg, "The Polycomb complex PRC2 and its mark in life," Nature, vol. 469, no. 7330, pp. 343-349, 2011.

[7] K. H. Kim and C. W. M. Roberts, "Targeting EZH2 in cancer," Nature Medicine, vol. 22, no. 2, pp. 128-134, 2016.

[8] M. Yamagishi and K. Uchimaru, "Targeting EZH2 in cancer therapy," Current Opinion in Oncology, vol. 29, no. 5, pp. 375-381, 2017.

[9] L. Gan, Y. Yang, Q. Li, Y. Feng, T. Liu, and W. Guo, "Epigenetic regulation of cancer progression by EZH2: from 
biological insights to therapeutic potential," Biomarker Research, vol. 6, no. 1, p. 10, 2018.

[10] D. Moher, L. Shamseer, M. Clarke et al., "Preferred reporting items for systematic review and meta-analysis protocols (PRISMA-P) 2015 statement," Systematic Reviews, vol. 4, no. 1, p. 1, 2015.

[11] J. F. Tierney, L. A. Stewart, D. Ghersi, S. Burdett, and M. R. Sydes, "Practical methods for incorporating summary timeto-event data into meta-analysis," Trials, vol. 8, no. 1, p. 16, 2007.

[12] Z. Li, L. Xu, N. Tang et al., "The polycomb group protein EZH2 inhibits lung cancer cell growth by repressing the transcription factor Nrf2," FEBS Letters, vol. 588, no. 17, pp. 30003007, 2014.

[13] C. Behrens, L. M. Solis, H. Lin et al., "EZH2 protein expression associates with the early pathogenesis, tumor progression, and prognosis of non-small cell lung carcinoma," Clinical Cancer Research, vol. 19, no. 23, pp. 6556-6565, 2013.

[14] X. Chen, N. Song, K. Matsumoto et al., "High expression of trimethylated histone $\mathrm{H} 3$ at lysine 27 predicts better prognosis in non-small cell lung cancer," International Journal of Oncology, vol. 43, no. 5, pp. 1467-1480, 2013.

[15] Huqun, R. Ishikawa, J. Zhang et al., "Enhancer of zeste homo$\log 2$ is a novel prognostic biomarker in nonsmall cell lung cancer," Cancer, vol. 118, no. 6, pp. 1599-1606, 2012.

[16] J. Kikuchi, I. Kinoshita, Y. Shimizu et al., "Distinctive expression of the polycomb group proteins Bmil polycomb ring finger oncogene and enhancer of zeste homolog 2 in nonsmall cell lung cancers and their clinical and clinicopathologic significance," Cancer, vol. 116, no. 12, pp. 3015-3024, 2010.

[17] M. Takawa, K. Masuda, M. Kunizaki et al., "Validation of the histone methyltransferase EZH2 as a therapeutic target for various types of human cancer and as a prognostic marker," Cancer Science, vol. 102, no. 7, pp. 1298-1305, 2011.

[18] C. Xu, K. Hao, H. Hu et al., "Expression of the enhancer of zeste homolog 2 in biopsy specimen predicts chemoresistance and survival in advanced non-small cell lung cancer receiving first-line platinum-based chemotherapy," Lung Cancer, vol. 86, no. 2, pp. 268-273, 2014.

[19] C. Xu, Z. Hou, P. Zhan et al., "EZH2 regulates cancer cell migration through repressing TIMP-3 in non-small cell lung cancer," Medical Oncology, vol. 30, no. 4, p. 713, 2013.

[20] A.-L. R. Ø. Bondgaard, T. T. Poulsen, H. S. Poulsen, and B. G. Skov, "Different expression of EZH2, BMI1 and Ki67 in low and high grade neuroendocrine tumors of the lung," Cancer Biomarkers, vol. 11, no. 2-3, pp. 123-128, 2012.

[21] J. Geng, X. Li, Z. Zhou, C. L. Wu, X. Bai, and M. Dai, “EZH2 promotes tumor progression via regulating VEGF-A/AKT signaling in non-small cell lung cancer," Cancer Letters, vol. 359, no. 2, pp. 275-287, 2015.

[22] G. Toyokawa, K. Takada, T. Okamoto et al., "Elevated metabolic activity on 18F-FDG PET/CT is associated with the expression of EZH2 in non-small cell lung cancer," Anticancer Research, vol. 37, no. 3, pp. 1393-1401, 2017.

[23] G. Toyokawa, K. Takada, T. Tagawa et al., "Prevalence of enhancer of zeste homolog 2 in patients with resected small cell lung cancer," Anticancer Research, vol. 38, no. 6, pp. 3707-3711, 2018.

[24] T. Matsubara, G. Toyokawa, K. Takada et al., "The association and prognostic impact of enhancer of zeste homologue 2 expression and epithelial-mesenchymal transition in resected lung adenocarcinoma," PLoS One, vol. 14, no. 5, article e0215103, 2019.

[25] S. Wu, D. Wu, Y. Pan, H. Liu, Z. Shao, and M. Wang, "Correlation between EZH2 and CEP55 and lung adenocarcinoma prognosis," Pathology, Research and Practice, vol. 215, no. 2, pp. 292-301, 2019.

[26] X. Wang, L. T. Brea, and J. Yu, "Immune modulatory functions of EZH2 in the tumor microenvironment: implications in cancer immunotherapy," American Journal of Clinical and Experimental Urology, vol. 7, no. 2, pp. 85-91, 2019.

[27] T. Jiang, Y. Wang, F. Zhou, G. Gao, S. Ren, and C. Zhou, "Prognostic value of high EZH2 expression in patients with different types of cancer: a systematic review with meta-analysis," Oncotarget, vol. 7, no. 4, pp. 4584-4597, 2016.

[28] X. Wang, H. Zhao, L. Lv, L. Bao, X. Wang, and S. Han, "Prognostic significance of EZH2 expression in non-small cell lung cancer: a meta-analysis," Scientific Reports, vol. 6, no. 1, article 19239, 2016.

[29] Z. Chen, X. Chen, B. Lu et al., "Up-regulated LINC01234 promotes non-small-cell lung cancer cell metastasis by activating VAV3 and repressing BTG2 expression," Journal of Hematology \& Oncology, vol. 13, no. 1, p. 7, 2020.

[30] X. Wang, H. Liu, K. Shen et al., "Long intergenic non-coding RNA 00467 promotes lung adenocarcinoma proliferation, migration and invasion by binding with EZH2 and repressing HTRA3 expression," Molecular Medicine Reports, vol. 20, no. 1, pp. 640-654, 2019.

[31] Z. Sun, C. He, M. Xiao et al., "LncRNA FOXC2 antisense transcript accelerates non-small-cell lung cancer tumorigenesis via silencing p15," American Journal of Translational Research, vol. 11, no. 7, pp. 4552-4560, 2019.

[32] K. Mozhui and A. K. Pandey, "Conserved effect of aging on DNA methylation and association with EZH2 polycomb protein in mice and humans," Mechanisms of Ageing and Development, vol. 162, pp. 27-37, 2017.

[33] T. Ito, Y. V. Teo, S. A. Evans, N. Neretti, and J. M. Sedivy, "Regulation of cellular senescence by polycomb chromatin modifiers through distinct DNA damage- and histone methylation-dependent pathways," Cell Reports, vol. 22, no. 13, pp. 3480-3492, 2018.

[34] G. Zhang, Y.-J. Wu, and F. Yan, "MicroRNA-130-5p promotes invasion as well as migration of lung adenocarcinoma cells by targeting the EZH2 signaling pathway," European Review for Medical and Pharmacological Sciences, vol. 23, no. 21, pp. 9480-9488, 2019.

[35] D. Qu, W.-W. Sun, L. Li et al., "Long noncoding RNA MALAT1 releases epigenetic silencing of HIV-1 replication by displacing the polycomb repressive complex 2 from binding to the LTR promoter," Nucleic Acids Research, vol. 47, no. 6, pp. 3013-3027, 2019.

[36] Y. Liu, Q. Hu, and X. Wang, "AFAP1-AS1 induces cisplatin resistance in non-small cell lung cancer through PI3K/AKT pathway," Oncology Letters, vol. 19, no. 1, pp. 1024-1030, 2020.

[37] X. Liu, X. Lu, F. Zhen et al., "LINC00665 induces acquired resistance to gefitinib through recruiting $\mathrm{EZH} 2$ and activating PI3K/AKT pathway in NSCLC," Molecular Therapy Nucleic Acids, vol. 16, pp. 155-161, 2019.

[38] J. Zhan, P. Wang, S. Li et al., "HOXB13 networking with ABCG1/EZH2/slug mediates metastasis and confers resistance to cisplatin in lung adenocarcinoma patients," Theranostics, vol. 9, no. 7, pp. 2084-2099, 2019. 
[39] C. R. Majer, L. Jin, M. P. Scott et al., “A687V EZH2 is a gain-offunction mutation found in lymphoma patients," FEBS Letters, vol. 586, no. 19, pp. 3448-3451, 2012.

[40] C. Baugé, C. Bazille, N. Girard, E. Lhuissier, and K. Boumediene, "Histone methylases as novel drug targets: developing inhibitors of EZH2," Future Medicinal Chemistry, vol. 6, no. 17, pp. 1943-1965, 2014.

[41] Y. Zhao, X.-X. Wang, W. Wu et al., "EZH2 regulates PD-L1 expression via HIF- $1 \alpha$ in non-small cell lung cancer cells," Biochemical and Biophysical Research Communications, vol. 517, no. 2, pp. 201-209, 2019.

[42] G.-Q. Wu, K.-Q. Chai, X.-M. Zhu et al., “Anti-cancer effects of curcumin on lung cancer through the inhibition of EZH2 and NOTCH1," Oncotarget, vol. 7, no. 18, pp. 26535-26550, 2016.

[43] A. E. Frankel, X. Liu, and J. D. Minna, "Developing EZH2targeted therapy for lung cancer," Cancer Discovery, vol. 6, no. 9, pp. 949-952, 2016.

[44] M. Saito, K. Saito, K. Shiraishi et al., "Identification of candidate responders for anti-PD-L1/PD-1 immunotherapy, RovaT therapy, or EZH2 inhibitory therapy in small-cell lung cancer," Molecular and Clinical Oncology, vol. 8, no. 2, pp. 310314, 2018.

[45] Q. Feng, H. He, T. Gao et al., "Synthesis and biological evaluation of benzomorpholine derivatives as novel EZH2 inhibitors for anti-non-small cell lung cancer activity," Molecular Diversity, vol. 23, no. 3, pp. 681-696, 2019.

[46] S. Huang, Z. Wang, J. Zhou et al., "EZH2 inhibitor GSK126 suppresses antitumor immunity by driving production of myeloid-derived suppressor cells," Cancer Research, vol. 79, no. 8, pp. 2009-2020, 2019.

[47] P. Goldstraw, K. Chansky, J. Crowley et al., "The IASLC lung cancer staging project: proposals for revision of the TNM stage groupings in the forthcoming (eighth) edition of the TNM classification for lung cancer," Journal of Thoracic Oncology, vol. 11, no. 1, pp. 39-51, 2016.

[48] G. A. Woodard, K. D. Jones, and D. M. Jablons, "Lung cancer staging and prognosis," Cancer Treatment and Research, vol. 170, pp. 47-75, 2016.

[49] O. Rena, F. Massera, M. Robustellini et al., "Use of the proposals of the international association for the study of lung cancer in the forthcoming edition of lung cancer staging system to predict long-term prognosis of operated patients," Cancer Journal, vol. 16, no. 2, pp. 176-181, 2010.

[50] W. H. Ban, C. D. Yeo, S. Han et al., "Impact of smoking amount on clinicopathological features and survival in nonsmall cell lung cancer," BMC Cancer, vol. 20, no. 1, p. 848, 2020.

[51] G. Anzalone, G. Arcoleo, F. Bucchieri et al., "Cigarette smoke affects the onco-suppressor DAB2IP expression in bronchial epithelial cells of COPD patients," Scientific Reports, vol. 9, no. 1, article 15682, 2019.

[52] M. Hussain, M. Rao, A. E. Humphries et al., "Tobacco smoke induces polycomb-mediated repression of Dickkopf-1 in lung cancer cells," Cancer Research, vol. 69, no. 8, pp. 3570-3578, 2009.

[53] T. Huang, X. Chen, Q. Hong et al., "Meta-analyses of gene methylation and smoking behavior in non-small cell lung cancer patients," Scientific Reports, vol. 5, no. 1, article 8897, 2015.

[54] Y. Guo, R. Cao, X. Zhang et al., "Recent progress in rare oncogenic drivers and targeted therapy for non-small cell lung cancer," Oncotargets and Therapy, vol. 12, pp. 10343-10360, 2019.
[55] P. J. Roberts and C. J. Der, "Targeting the Raf-MEK-ERK mitogen-activated protein kinase cascade for the treatment of cancer," Oncogene, vol. 26, no. 22, pp. 3291-3310, 2007.

[56] R. H. J. Breuer, P. J. F. Snijders, E. F. Smit et al., "Increased expression of the EZH2 polycomb group gene in BMI-1positive neoplastic cells during bronchial carcinogenesis," Neoplasia, vol. 6, no. 6, pp. 736-743, 2004.

[57] S. Varambally, S. M. Dhanasekaran, M. Zhou et al., "The polycomb group protein EZH2 is involved in progression of prostate cancer," Nature, vol. 419, no. 6907, pp. 624-629, 2002.

[58] G. Nikoloski, S. M. C. Langemeijer, R. P. Kuiper et al., "Somatic mutations of the histone methyltransferase gene EZH2 in myelodysplastic syndromes," Nature Genetics, vol. 42, no. 8, pp. 665-667, 2010.

[59] X. Wang, L. Sehgal, N. Jain, T. Khashab, R. Mathur, and F. Samaniego, "LncRNA MALAT1 promotes development of mantle cell lymphoma by associating with EZH2," Journal of Translational Medicine, vol. 14, no. 1, p. 346, 2016.

[60] M. T. McCabe, H. M. Ott, G. Ganji et al., "EZH2 inhibition as a therapeutic strategy for lymphoma with EZH2-activating mutations," Nature, vol. 492, no. 7427, pp. 108-112, 2012.

[61] R. Schmitz, G. W. Wright, D. W. Huang et al., "Genetics and pathogenesis of diffuse large B-cell lymphoma," The New England Journal of Medicine, vol. 378, no. 15, pp. 1396-1407, 2018. 